\title{
Can pathoanatomical pathways of degeneration in lumbar motion segments be identified by clustering MRI findings
}

Rikke K Jensen ${ }^{1,2^{*}}$, Tue S Jensen ${ }^{1,2}$, Per Kjaer ${ }^{1,3}$ and Peter Kent ${ }^{1,2}$

\begin{abstract}
Background: Magnetic Resonance Imaging (MRI) is the gold standard for detailed visualisation of spinal pathological and degenerative processes, but the prevailing view is that such imaging findings have little or no clinical relevance for low back pain. This is because these findings appear to have little association with treatment effects in clinical populations, and mostly a weak association with the presence of pain in the general population. However, almost all research into these associations is based on the examination of individual MRI findings, despite its being very common for multiple MRI findings to coexist. Therefore, this proof-of-concept study investigated the capacity of a multivariable statistical method to identify clusters of MRI findings and for those clusters to be grouped into pathways of vertebral degeneration.

Methods: This study is a secondary analysis of data from 631 patients, from an outpatient spine clinic, who had been screened for inclusion in a randomised controlled trial. The available data created a total sample pool of 3,155 vertebral motion segments. The mean age of the cohort was 42 years (SD 10.8, range 18-73) and 54\% were women. MRI images were quantitatively coded by an experienced musculoskeletal research radiologist using a detailed and standardised research MRI evaluation protocol that has demonstrated high reproducibility. Comprehensive MRI findings descriptive of the disco-vertebral component of lumbar vertebrae were clustered using Latent Class Analysis. Two pairs of researchers, each containing an experienced MRI researcher, then independently categorised the clusters into hypothetical pathoanatomic pathways based on the known histological changes of discovertebral degeneration.

Results: Twelve clusters of MRI findings were identified, described and grouped into five different hypothetical pathways of degeneration that appear to have face validity.

Conclusions: This study has shown that Latent Class Analysis can be used to identify clusters of MRI findings from people with LBP and that those clusters can be grouped into degenerative pathways that are biologically plausible. If these clusters of MRI findings are reproducible in other datasets of similar patients, they may form a stable platform to investigate the relationship between degenerative pathways and clinically important characteristics such as pain and activity limitation.
\end{abstract}

Keywords: Low back pain, Disc degeneration, Magnetic Resonance Imaging, Subgroup, Latent Class Analysis

\footnotetext{
* Correspondence: rikke.kruger.jensen@rsyd.dk

${ }^{1}$ Research Department, Spine Centre of Southern Denmark, Hospital

Lillebaelt, Middelfart, Denmark

${ }^{2}$ Institute of Regional Health Services Research, University of Southern

Denmark, Middelfart, Denmark

Full list of author information is available at the end of the article
} 


\section{Background}

While it is widely accepted that low back pain (LBP) is a bio-psycho-social condition, concern has recently been expressed that LBP research is moving too far from the biological component [1]. However, despite the large body of previous research attempting to identify specific biological reasons for LBP, there are still more questions than answers. The clinical consequence of this uncertainty is that only approximately $15 \%$ of primary care patients with LBP are classified with a specific pathoanatomic diagnosis, with the remaining $85 \%$ being diagnosed with nonspecific LBP [2].

In research, non-specific LBP is often conceptualised as one condition, although most clinicians and researchers believe that non-specific LBP is actually a heterogenous mix of conditions [3]. This distinction occurs because, although many methods to subgroup non-specific LBP have been proposed, the evidence for the validity of almost all these approaches remains very tentative [4]. It is also the case that almost all subgrouping methods that have demonstrated any treatment validity are not based on pathoanatomy [5], the exception being the established association between pain centralisation and discogenic pain [6,7].

One way of categorising patients into diagnostic subgroups is by pathoanatomy identified with Magnetic Resonance Imaging (MRI). MRI has increasingly replaced other imaging modalities in the diagnosis of LBP because of the level of detail it provides. Paradoxically, despite the unprecedented level of detail available on spinal pathological or degenerative findings, the prevailing view is that they have little clinical relevance [8-10]. This is because these findings seem to have little or no association with treatment effect [11] in clinical populations, and mostly a weak and inconsistent association with the presence of pain in the general population [12]. Furthermore, when using MRI, there is normally no way to distinguish age-related degeneration (believed to be non-painful) and 'pathological' or trauma-related degeneration (believed to be painful). Similarly, it has been reported that normal aging of the motion segment and pathological degeneration cannot be distinguished at a histological level [13], suggesting that the biological process is the same in normal aging but is accelerated in the pathological state. Therefore, the biological age of a vertebral motion segment is not necessarily the same as the actual chronological age of the person.

However, investigation into associations between MRI findings and pain are complicated by numerous MRI findings being present at the same time. For example, vertebral endplate signal changes and vertebral disc herniations almost always co-exist with other degenerative disc findings, such as reduction of height and reduction of the signal intensity of the disc [14-17]. Despite this, most previous research has focused solely on the association between single imaging findings and pain or other clinical outcomes. It is only recently that researchers have started to explore the potential for answers within this complexity, for example Cheung and co-workers[18], who have reported a positive correlation between the sum of degenerative disc MRI findings and low back pain.

Classification systems exist for categorising the detailed information on the physiological condition of a motion segment that MRI provides. For example, the Pfirrmann classification [19] is a descriptive tool for classifying intervertebral disc degeneration. However, such classification systems also typically focus on findings related to one or two anatomic entities, such as the height and signal intensity of the intervertebral disc, leaving un-described other relevant entities such as vertebral endplate irregularity, vertebral endplate signal changes and disc herniations.

It would be useful if there were methods to better model the multivariable relationships between clusters of MRI findings, as these might provide a clearer understanding of how pathoanatomical processes are expressed across all the structures associated with a vertebral segment. Furthermore, the identification of such processes, and an ability of such clusters to stage the biological age of a vertebral segment, might allow better insight into the relationship between degenerative processes and clinical characteristics such as pain and activity limitation.

Therefore, the aims of this study were to investigate: (i) how multiple MRI findings from lumbar spine motion segments cluster together in patients with chronic LBP, and (ii) to classify these clusters of MRI findings into hypothetical pathoanatomical pathways.

\section{Methods}

This was a 'proof of concept' study designed to test a novel analytical method and set hypotheses about pathoanatomical pathways of vertebral segment degeneration. It used cross-sectional data and a probabilistic (Bayesian) Latent Class Analysis approach to the multivariable clustering of MRI findings.

\section{Study sample}

This study is a secondary analysis of data from a cohort of patients who were potential participants in a randomised controlled trial [20]. All participants attended the same publically-funded outpatient spine clinic (The Spine Centre of Southern Denmark) where they had been referred from the primary care sector for a multidisciplinary evaluation. In this clinical setting, from June 2006 to June 2008, MRI was routinely performed on all patients (who had no contraindications for MRI) who met the following criteria: (a) LBP or leg pain of at least 3 on an 11-point Numerical Rating Scale, (b) duration of current symptoms from 2 to 12 months, and (c) age above 18 years. 


\section{Data collection}

MRI was performed with a $0.2 \mathrm{~T}$ MRI-system (Magnetom Open Viva; Siemens AG, Erlangen, Germany). A body spine surface coil was used for imaging of the lumbar region, with the study subjects in the supine position. The imaging protocol consisted of the following sequences:

- Localizer sequence, 40/10/40 (TR/TE/flip angle), two coronal and three sagittal images in orthogonal planes

- Sagittal T1-weighted spin echo, 621/26 (TR/TE), 144 x 256 matrix, $300 \mathrm{~mm}$ FOV, and 11 slices $4 \mathrm{~mm}$ wide, distance factor 0.20 .

- Sagittal T2-weighted turbo spin echo, 4609/134 (TR/effective TE), 210 x 256 matrix, 300 mm FOV, and 11 slices $4 \mathrm{~mm}$ wide, distance factor 0.20 .

- Axial T1-weighted spin echo, 720/26 (TR/TE), $192 \times$ 256 matrix, $240 \mathrm{~mm}$ FOV, and 15 slices $5 \mathrm{~mm}$ wide, distance factor 0.25 .

- Axial T2-weighted turbo spin echo, 6415/134 (TR/ effective TE), 180 x 256 matrix, $250 \mathrm{~mm}$ FOV, and 15 slices $5 \mathrm{~mm}$ wide, distance factor 0.25 .

- Axial images were performed on the three lower lumbar levels. If herniations were present at higher lumbar levels, relevant supplementing axial series were performed.

The images were quantitatively coded using a detailed and standardised research MRI evaluation protocol $[21,22]$. The evaluation was performed by an experienced musculoskeletal research radiologist who was blinded to any participant information other than name, age and sex.

\section{Variables of interest}

The following MRI variables were included in the current study: intervertebral disc height, disc signal intensity, disc herniations, size and type of vertebral endplate signal changes (VESC), irregularity of the vertebral endplate, osteophytes and spondylolisthesis. Each MRI variable was coded on two to seven different pathoanatomical stages (categories), as detailed in Additional file 1.

Previous testing of this MRI evaluation protocol when used by the same radiologist has shown substantial to almost perfect inter- and intra-observer reliability for the VESC variables (Kappa ranging from 0.73 to 1.0 ), and moderate to substantial reliability for other endplate related findings (Kappa ranging from 0.52 to 0.72) [21]. Evaluation of intervertebral disc-related changes has shown moderate to almost perfect reliability (Kappa ranging from 0.59 to 0.97 ) [22]. Anterolisthesis was evaluated according to Meyerding classification system [23].

\section{Statistical analyses}

MRI findings were clustered using the multivariable statistical program 'SNOB' (Monash Data Mining Centre,
Monash University, Melbourne, Victoria, Australia), which is a form of Latent Class Analysis. SNOB uses probabilistic mixture modelling and the minimum message length principle to determine the optimum class structure (clusters) within the data. Minimum message length is an automated statistical method to determine which of the possible models of class structure and explained variance are the most parsimonious and explanatory. Latent Class Analysis has a number of advantages over traditional cluster analysis techniques, including: greater classification accuracy, the ability to manage variables of all data types (dichotomous, ordinal and continuous), and a tolerance of missing data [24-26].

Analysis occurred at the level of individual vertebral segments, as lumbar segments within an individual person may each have a different 'pathoanatomical age'. Therefore, every participating person contributed five lumbar vertebral segments to the analysis.

The Latent Class Analysis identified the optimal number of clusters and also the cluster membership for each vertebral segment. We used this information to determine the proportion of vertebral segments within each cluster that displayed each coding category on each MRI variable and the proportion of vertebral levels (L1/2, L2/ 3 , L3/4 L4/5 and L5/S1) within each cluster. These data were calculated and graphed using Excel 2008 for Mac version 12.2.8 (Microsoft Corporation, Redmond, WA, USA).

Then two pairs of researchers, each containing an experienced MRI researcher, independently categorised the clusters into hypothetical pathoanatomic pathways based on the face validity of known histological changes of discovertebral degeneration. These histological changes had been identified by an (unpublished) electronic literature search (PubMed and Medline) and review. The pathways categorised by the pairs of researchers were compared and any differences resolved by discussion.

A post-hoc calculation of the mean (SD) chronological age of the motion segments within each cluster was also performed. This metric was used to test if the chronological age of each cluster challenged or supported the concept of 'pathoanatomical age' inherent in the pathways.

\section{Ethics}

This analysis was based on existing data collected for a randomised controlled trial[20] approved by the Ethics Committee for the Region of Southern Denmark (approval \# S-VF-20060111), registered in ClinicalTrials.gov (Identifier \# NCT00454792) and performed following the Declaration of Helsinki principles.

\section{Results}

Population

MRI findings and basic demographic data were available on 631 patients who had been screened for inclusion in 
the original studies. All were included in the current study cohort, which created a total sample pool of 3,155 vertebral motion segments. The mean age of the cohort was 42 years (SD 10.8, range 18-73) and 54\% were women. Detailed descriptive data of the MRI findings at a whole group level are tabulated in Additional file 1.

\section{Clusters of MRI findings}

The Latent Class Analysis identified twelve clusters of MRI findings. One cluster, characterised by no abnormal MRI findings, contained $52 \%$ of the 3,155 motion segments and represented the normal, pre-degenerative state. The second largest cluster contained almost 15\% of the motion segments and was characterised by motion segments with reduced disc height, reduced disc signal intensity, disc bulges and a minor degree of disc protrusions and high intensity zones. Sixty percent of these changes were located at the two lowest spinal levels. The rest of the clusters were smaller, with each containing less than $8 \%$ of the motion segments. Each cluster and its distribution of MRI findings is shown diagrammatically in Figures 1, 2, 3, 4, 5, 6, 7, 8, 9, 10, 11, and 12. More detailed descriptive data of the MRI findings at a cluster level are tabulated in Additional file 2.

\section{Hypothetical pathoanatomic pathways}

The hypothetical pathoanatomic pathways derived from the content analysis of the clusters were: (i) two clusters representing progressive stages of disc degeneration in the lower lumbar levels; (ii) four clusters representing progressive stages of disc herniations and VESC in the lower lumbar levels; (iii) two clusters containing progressive endplate changes and disc degeneration at the upper lumbar levels only; (iv) two cluster with VESC only, one cluster each for changes in the upper and the lower endplates; and lastly, (v) one cluster containing osteophytes at the upper lumbar motion segments. The pathoanatomic pathways and their clusters are illustrated in Figure 13.

Once the pathoanatomic pathways had been categorised, the mean age of the motion segments in each cluster was added to Figure 13 as a post-hoc analysis. The average age of the vertebral segments within each cluster supported the notion of a developmental model, as the chronological age in each cluster consistently increased across each pathoanatomic pathway as degeneration developed. Although the chronological age and biological age of vertebral segments may not be the same due to pathological degeneration, the observed age progression across the biological pathways adds credibility to the construct validity of this classification.

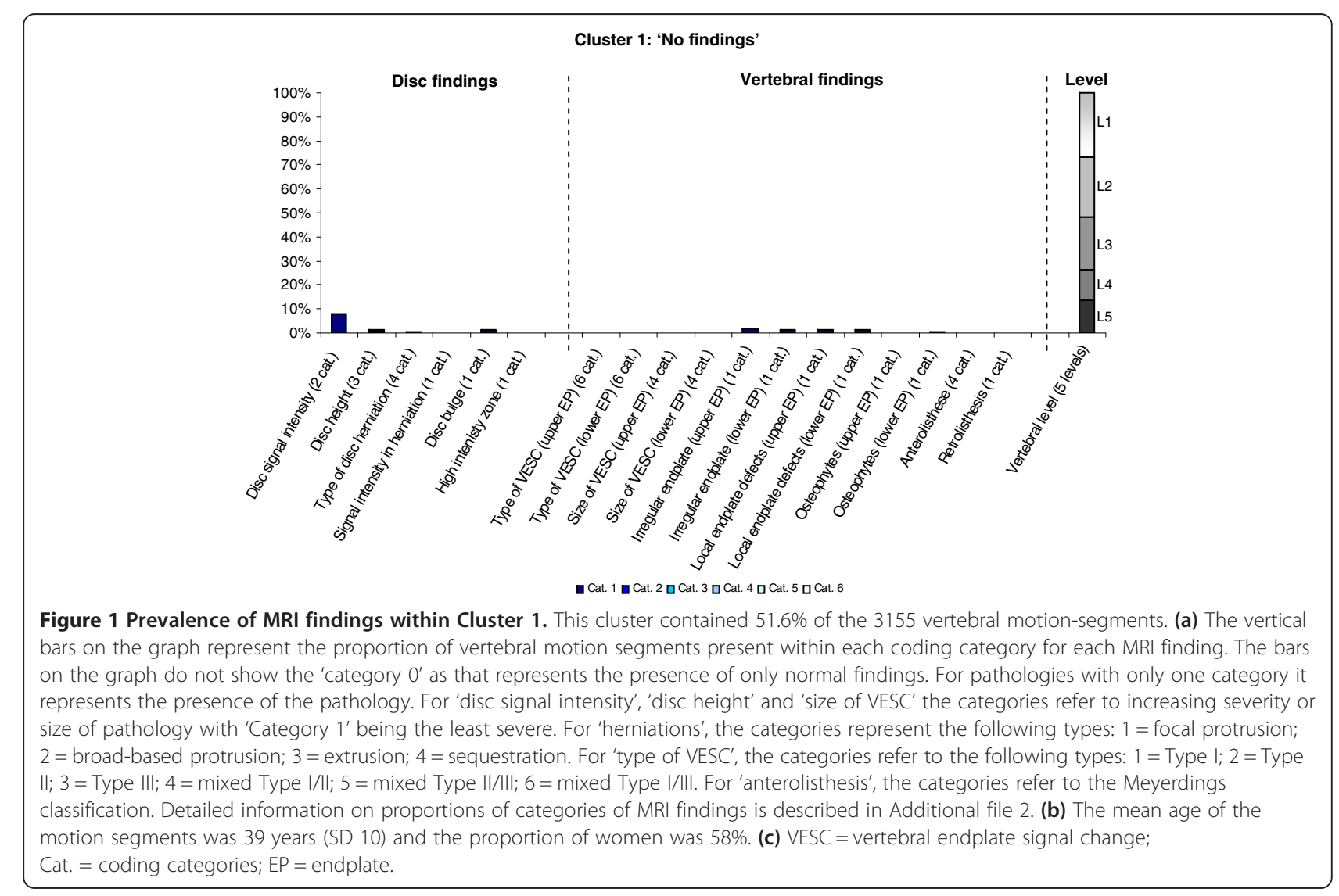




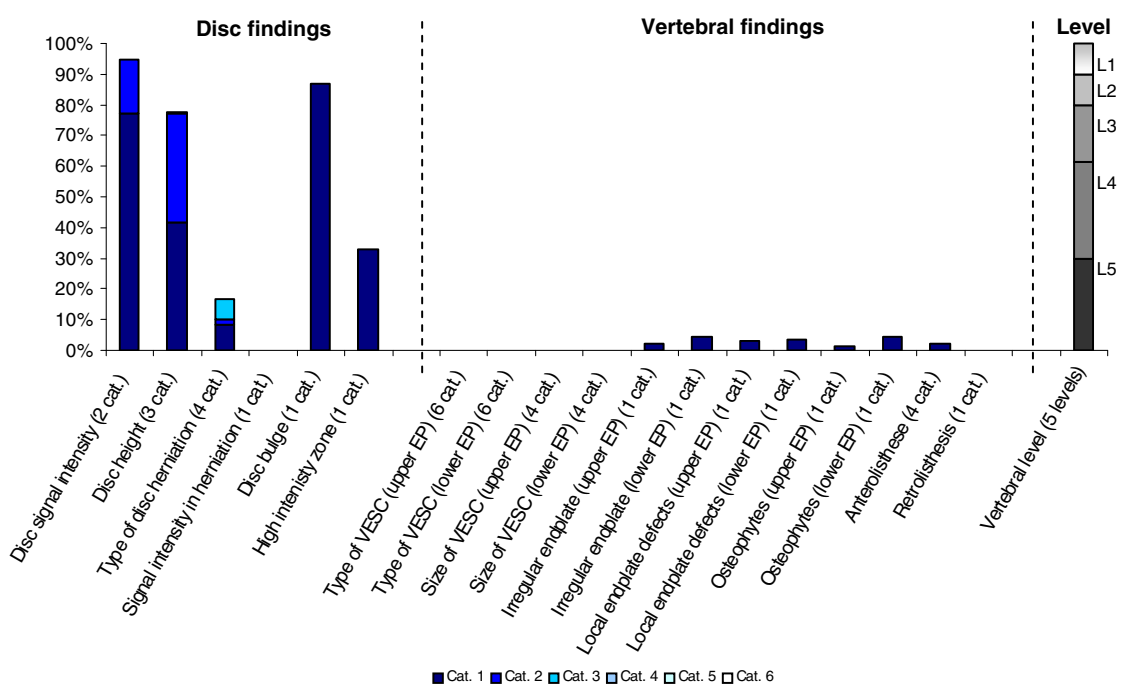

Figure 2 Proportion of categories of MRI findings for Cluster 2 containing 14.9\% of the $\mathbf{3 1 5 5}$ motion-segments. (a) The vertical bars on the graph represent the proportion of vertebral motion segments present within each coding category for each MRI finding. The bars on the graph do not show the 'Category 0', as that represents the presence of only normal findings. For pathologies with only one category, it represents the presence of the pathology. For 'disc signal intensity', 'disc height' and 'size of VESC' the categories refer to increasing severity or size of pathology with 'Category 1 ' being the least severe. For 'herniations', the categories represent the following types: $1=$ focal protrusion; 2 = broad-based protrusion; 3 = extrusion; 4 = sequestration. For 'type of $\mathrm{EESC}^{\prime}$ ', the categories refer to the following types: $1=$ Type I; $2=$ Type II; $3=$ Type $I I I 4=$ mixed Type I/II; $5=$ mixed Type I/III; $6=$ mixed Type I/III. For 'anterolisthesis' the categories refer to the Meyerdings classification. Detailed information on proportions of categories of MRI findings is described in Additional file 2. (b) The mean age of the motion segments was 46 years (SD 11) and the proportion of women was 55\%. (c) VESC = vertebral endplate signal change; Cat. = categories; $E P=$ endplate.

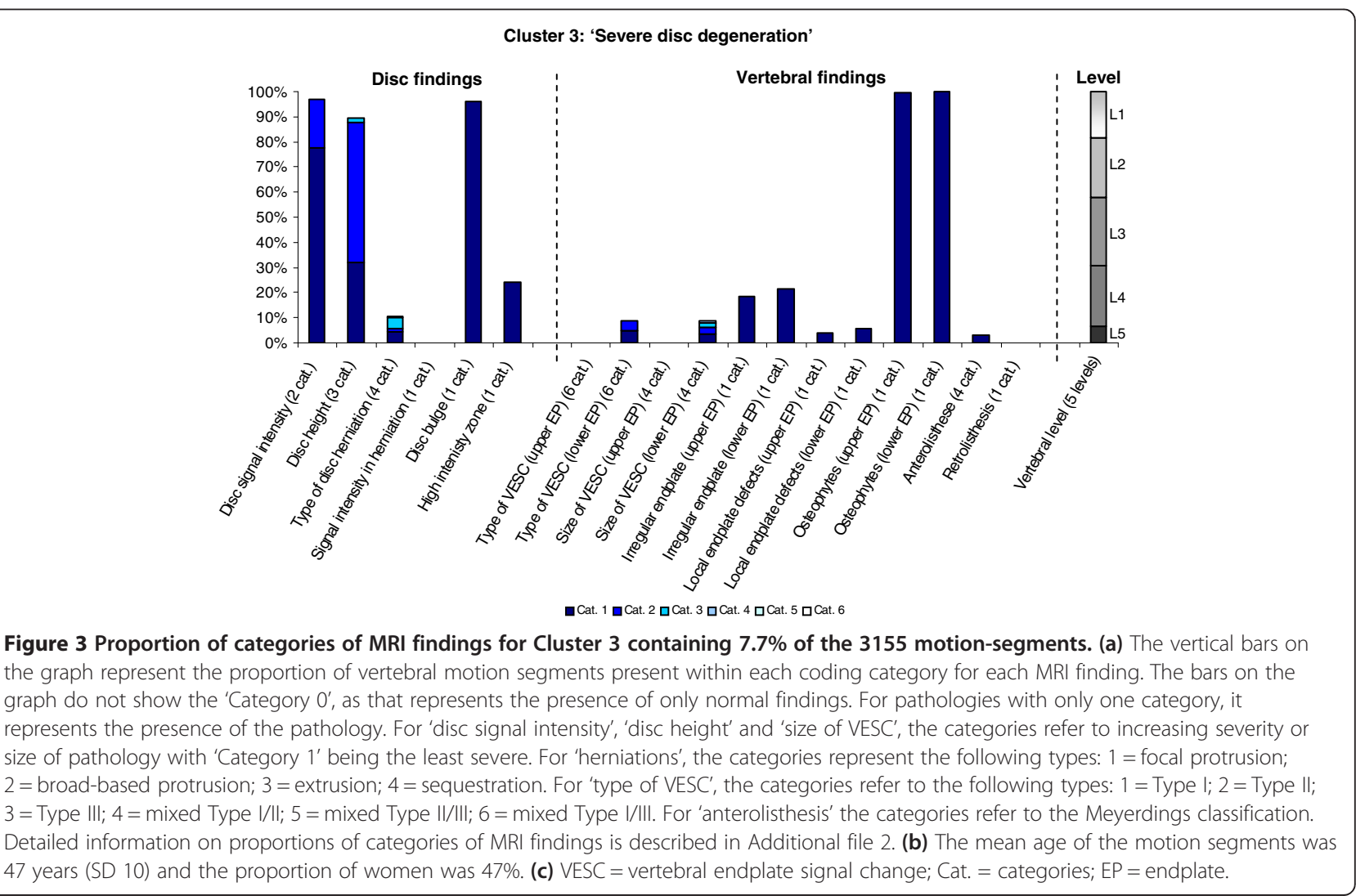




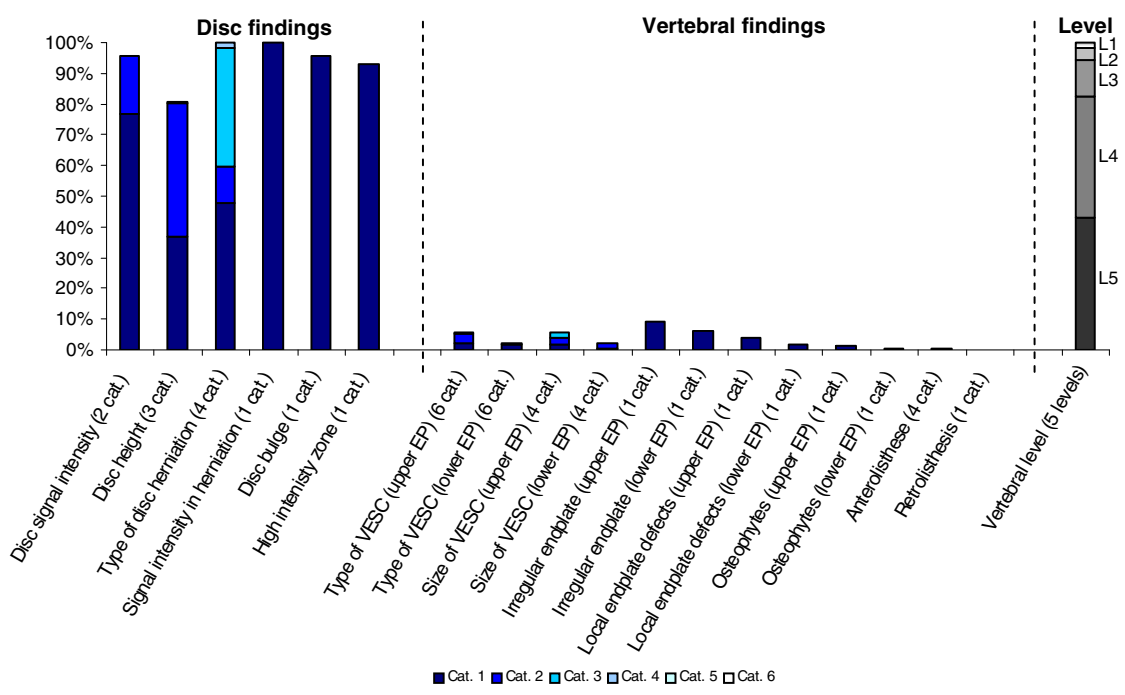

Figure 4 Proportion of categories of MRI findings for Cluster 4 containing 5.5\% of the $\mathbf{3 1 5 5}$ motion-segments. (a) The vertical bars on the graph represent the proportion of vertebral motion segments present within each coding category for each MRI finding. The bars on the graph do not show the 'Category 0', as that represents the presence of only normal findings. For pathologies with only one category, it represents the presence of the pathology. For 'disc signal intensity', 'disc height' and 'size of VESC', the categories refer to increasing severity or size of pathology with 'Category 1 ' being the least severe. For 'herniations', the categories represent the following types: $1=$ focal protrusion; 2 = broad-based protrusion; $3=$ extrusion; $4=$ sequestration. For 'type of VESC' the categories refer to the following types: $1=$ Type I; $2=$ Type II; 3 =Type III; $4=$ mixed Type I/II; $5=$ mixed Type I/III; $6=$ mixed Type I/III. For 'anterolisthesis', the categories refer to the Meyerdings classification. Detailed information on proportions of categories of MRI findings is described in Additional file 2. (b) The mean age of the motion segments was 41 years (SD 10) and the proportion of women was 48\%. (c) VESC = vertebral endplate signal change; Cat. = categories; $E P=$ endplate.

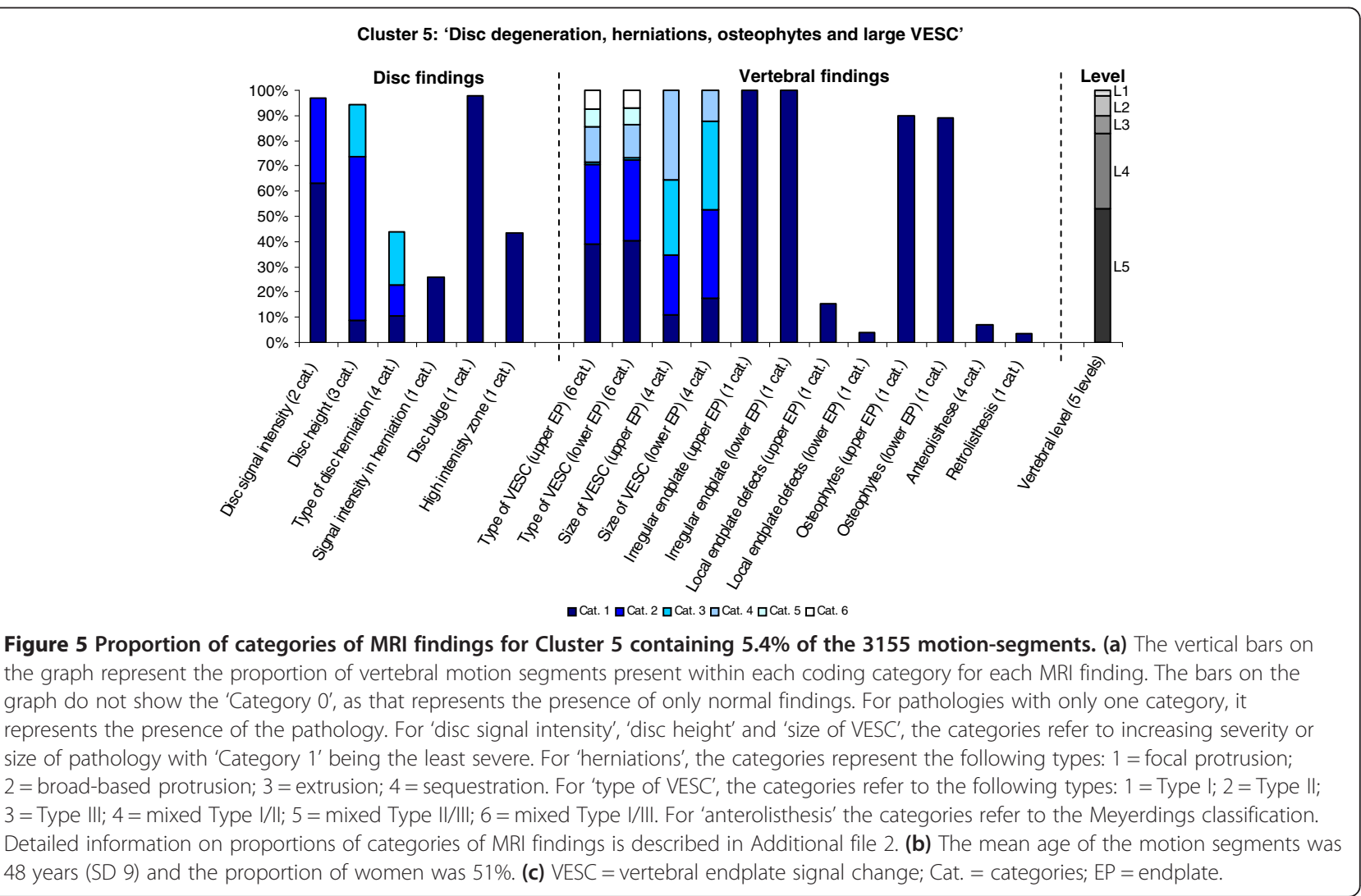




\section{Cluster 6: 'Disc degeneration, herniations, osteophytes and small VESC'}

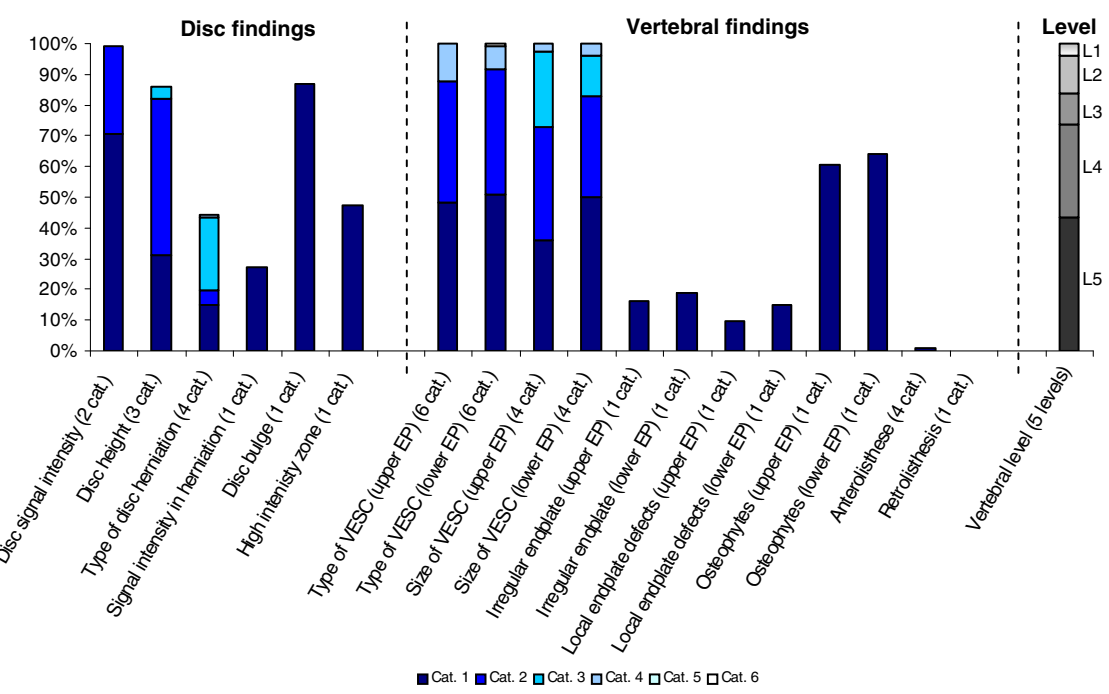

Figure 6 Proportion of categories of MRI findings for Cluster 6 containing 3.3\% of the $\mathbf{3 1 5 5}$ motion-segments. (a) The vertical bars on the graph represent the proportion of vertebral motion segments present within each coding category for each MRI finding. The bars on the graph do not show the 'Category 0 ' as that represents the presence of only normal findings. For pathologies with only one category, it represents the presence of the pathology. For 'disc signal intensity', 'disc height' and 'size of VESC', the categories refer to increasing severity or size of pathology with 'Category 1 ' being the least severe. For 'herniations', the categories represent the following types: $1=$ focal protrusion; 2 = broad-based protrusion; 3 =extrusion; $4=$ sequestration. For 'type of VESC', the categories refer to the following types: $1=$ Type l; $2=$ Type II; 3 =Type III; 4 = mixed Type I/II; 5 = mixed Type II/II; 6 = mixed Type I/III. For 'anterolisthesis', the categories refer to the Meyerdings classification. Detailed information on proportions of categories of MRI findings is described in Additional file 2. (b) The mean age of the motion segments was 47 years (SD 9) and the proportion of women was 53\%. (c) VESC = vertebral endplate signal change; Cat. = categories; EP = endplate.

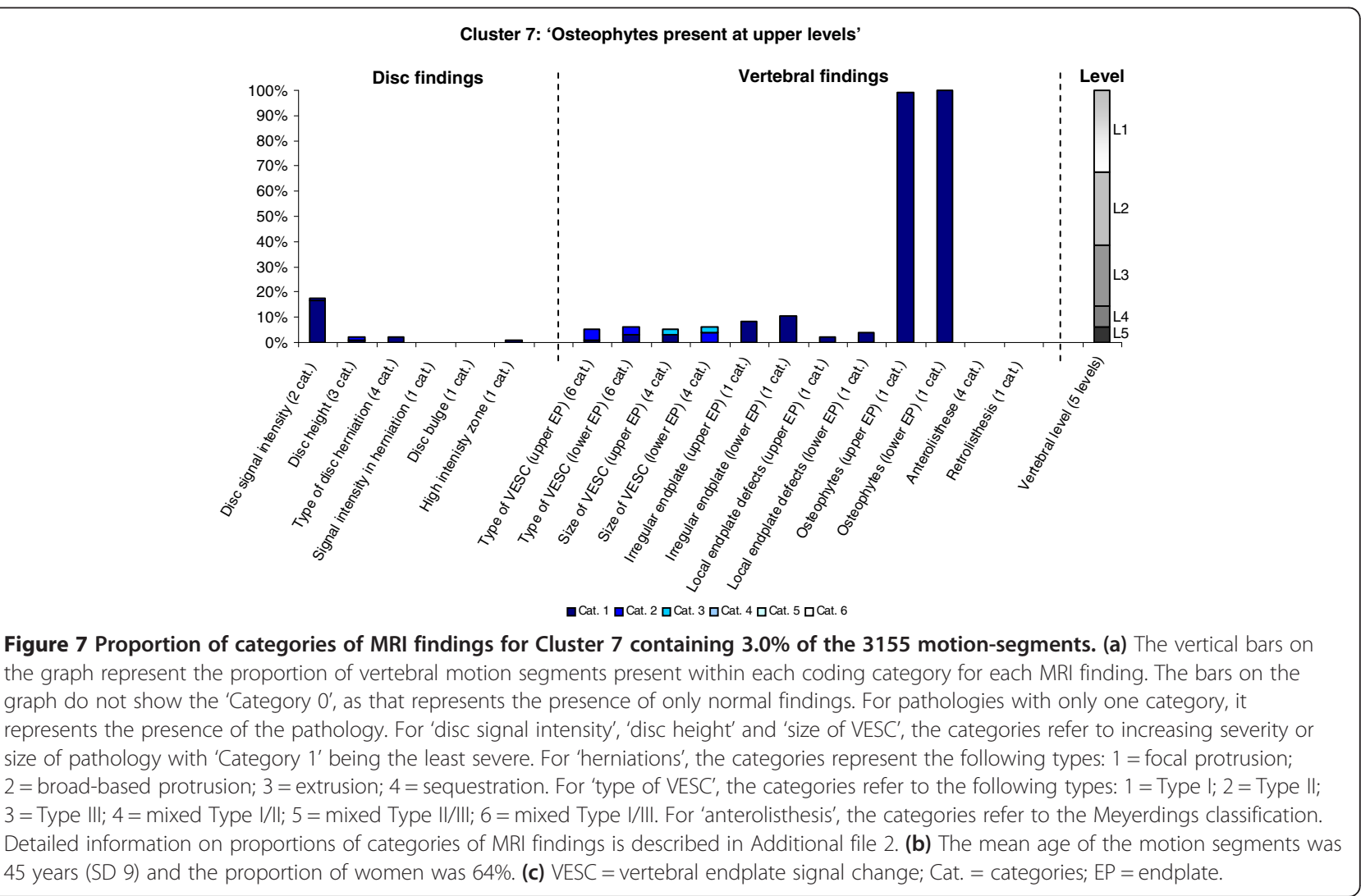




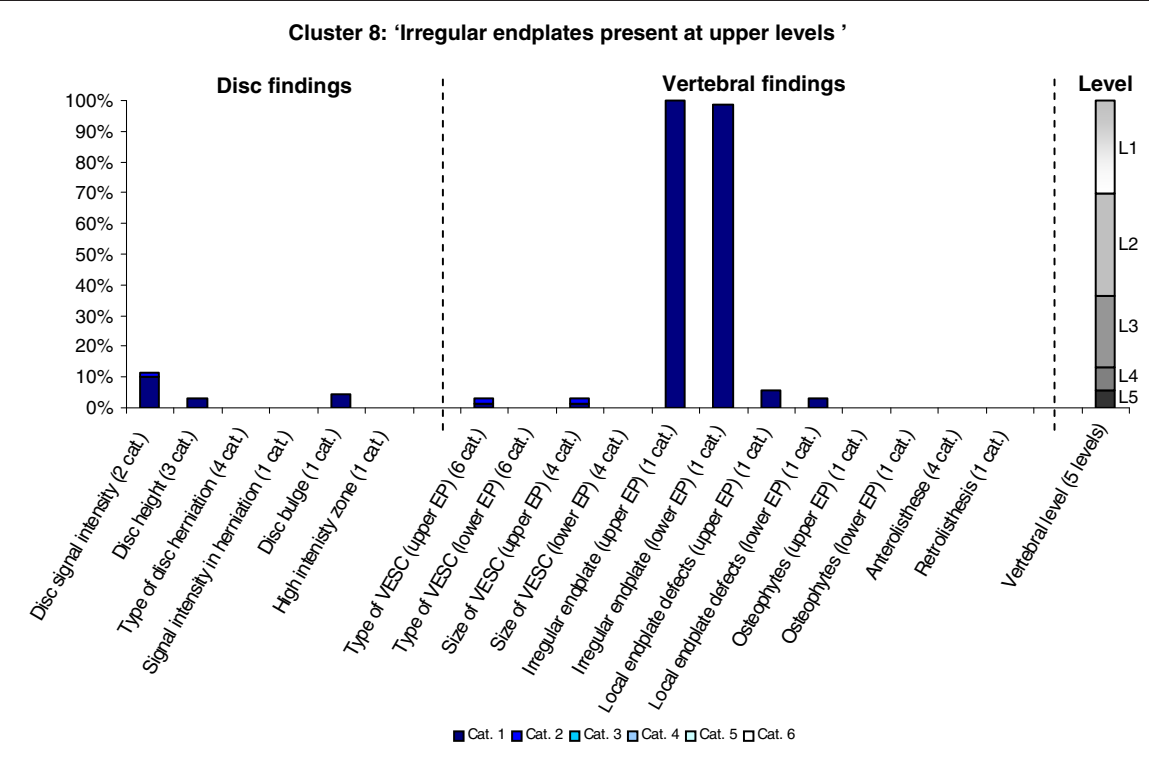

Figure 8 Proportion of categories of MRI findings for Cluster 8 containing 2.1\% of the $\mathbf{3 1 5 5}$ motion-segments. (a) The vertical bars on the graph represent the proportion of vertebral motion segments present within each coding category for each MRI finding. The bars on the graph do not show the 'Category $0^{\prime}$ as that represents the presence of only normal findings. For pathologies with only one category, it represents the presence of the pathology. For 'disc signal intensity', 'disc height' and 'size of VESC', the categories refer to increasing severity or size of pathology with 'Category 1 ' being the least severe. For 'herniations', the categories represent the following types: $1=$ focal protrusion; 2 = broad-based protrusion; 3 = extrusion; $4=$ sequestration. For 'type of $\mathrm{VESC}^{\prime}$ ', the categories refer to the following types: $1=$ Type I; $2=$ Type $I$; $3=$ Type III; $4=$ mixed Type I/II; $5=$ mixed Type II/II; $6=$ mixed Type I/III. For 'anterolisthesis', the categories refer to the Meyerdings classification. Detailed information on proportions of categories of MRI findings is described in Additional file 2. (b) The mean age of the motion segments was 35 years (SD 9) and the proportion of women was 28\%. (c) VESC = vertebral endplate signal change; Cat. = categories; EP = endplate.

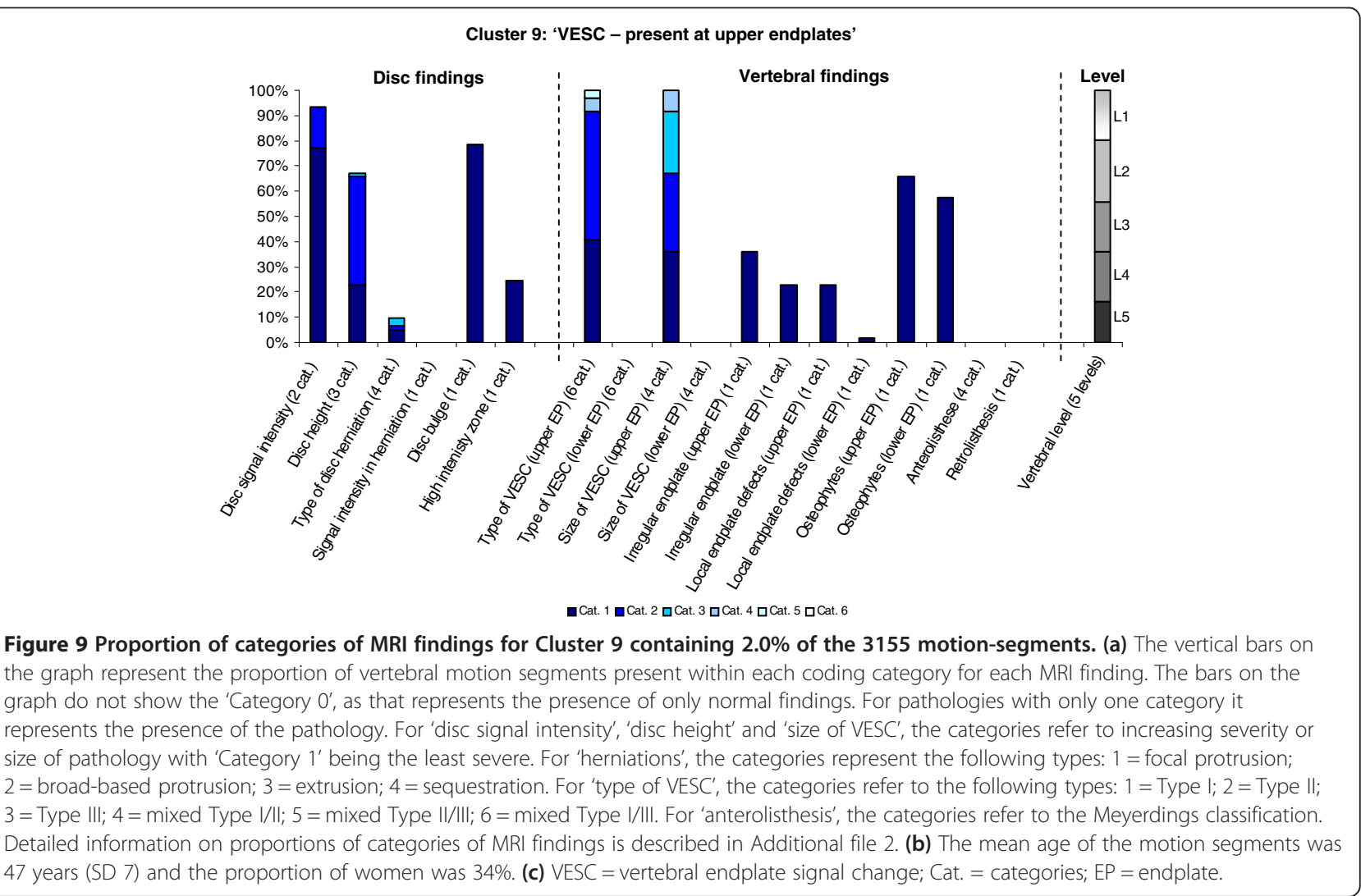




\section{Cluster 10: 'Disc degeneration, herniations and osteophytes'}

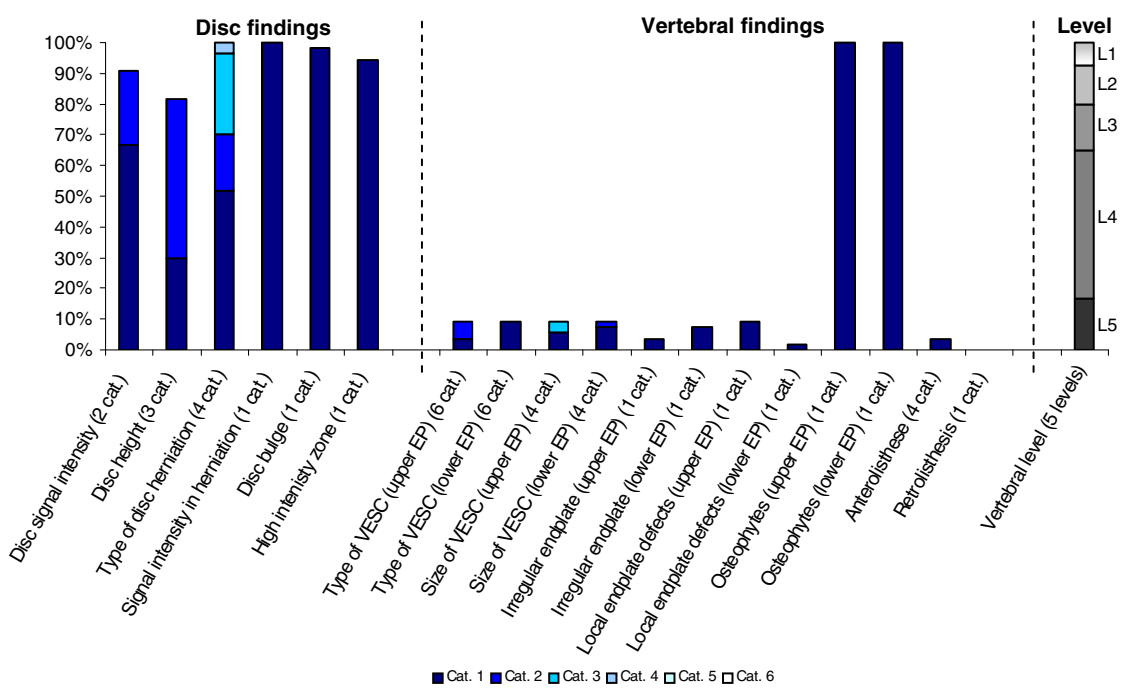

Figure 10 Proportion of categories of MRI findings for Cluster 10 containing $\mathbf{1 . 7 \%}$ of the $\mathbf{3 1 5 5}$ motion-segments. (a) The vertical bars on the graph represent the proportion of vertebral motion segments present within each coding category for each MRI finding. The bars on the graph do not show the 'Category 0', as that represents the presence of only normal findings. For pathologies with only one category it represents the presence of the pathology. For 'disc signal intensity', 'disc height' and 'size of VESC', the categories refer to increasing severity or size of pathology with 'Category 1 ' being the least severe. For 'herniations', the categories represent the following types: $1=$ focal protrusion; 2 = broad-based protrusion; 3 = extrusion; $4=$ sequestration. For 'type of VESC', the categories refer to the following types: $1=$ Type l; $2=$ Type II; $3=$ Type III; 4 = mixed Type I/II; $5=$ mixed Type II/III; $6=$ mixed Type I/III. For 'anterolisthesis', the categories refer to the Meyerdings classification. Detailed information on proportions of categories of MRI findings is described in Additional file 2. (b) The mean age of the motion segments was 43 years (SD 10) and the proportion of women was 50\%. (c) VESC = vertebral endplate signal change; Cat. = categories; $E P=$ endplate.

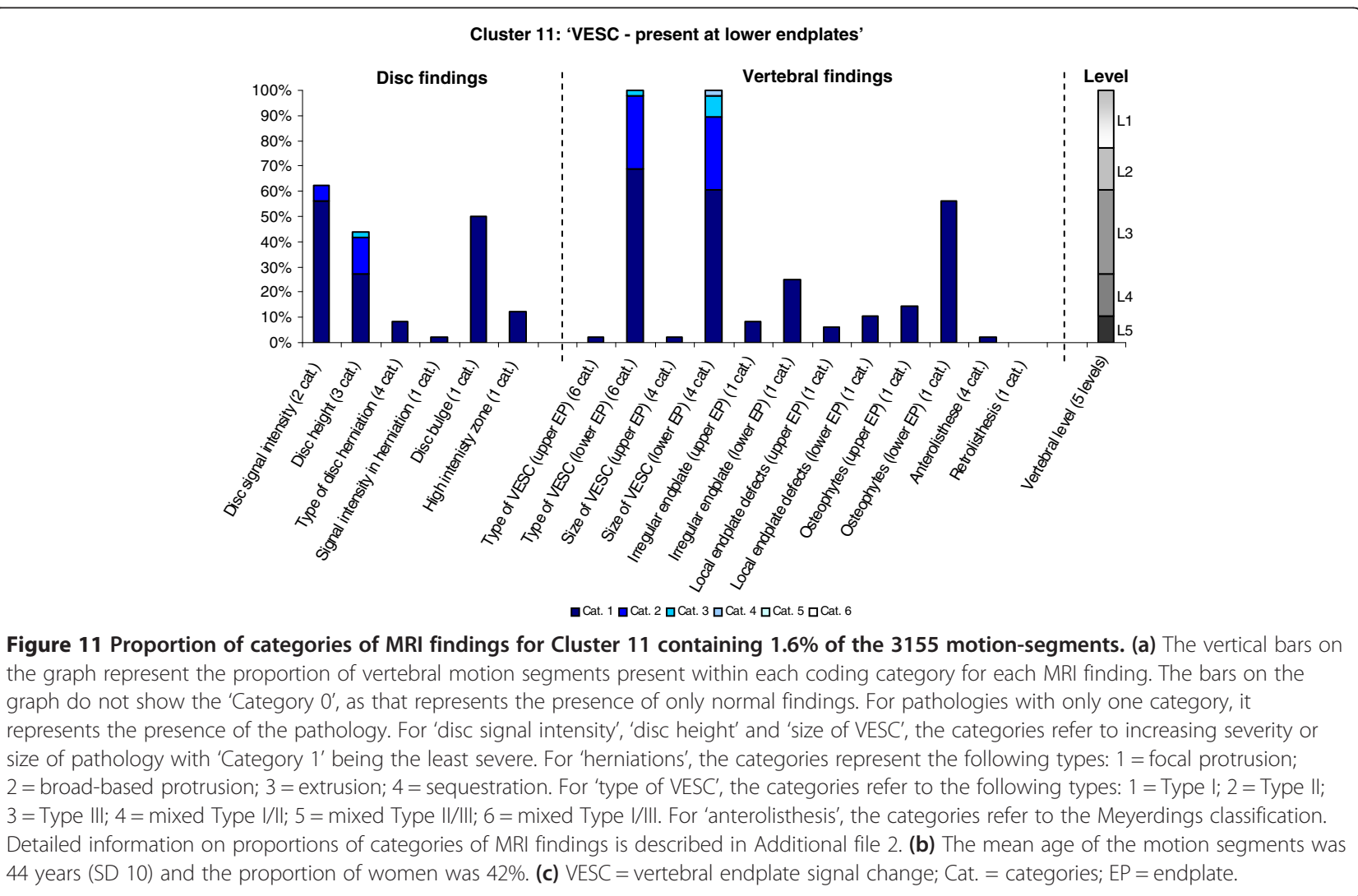




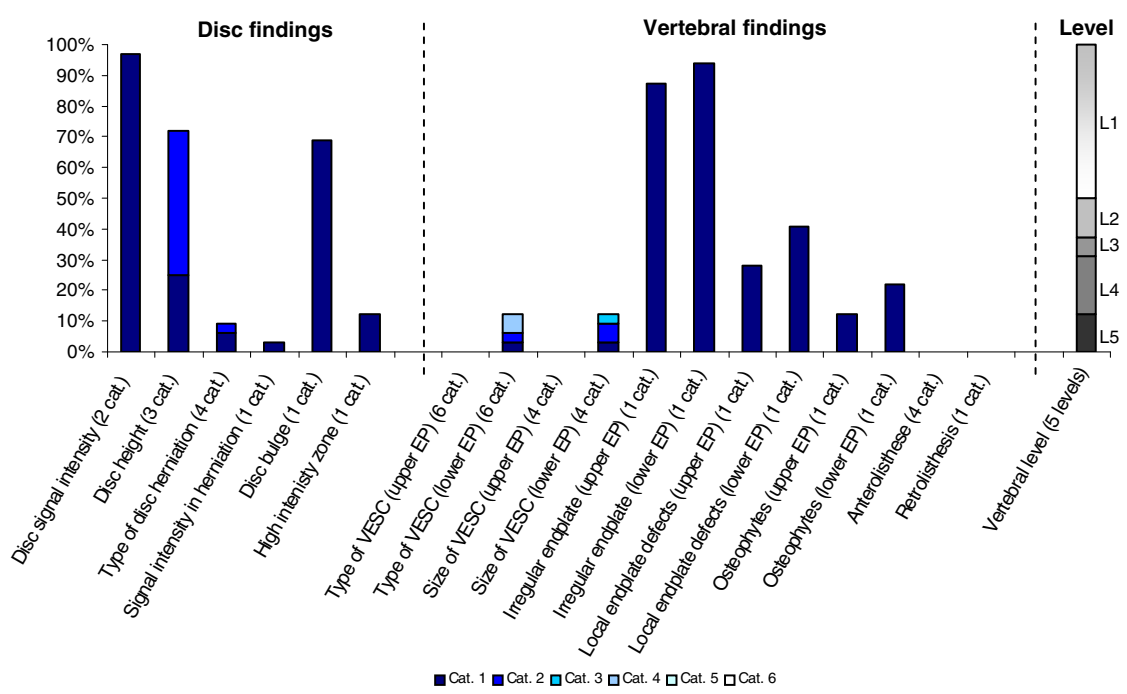

Figure 12 Proportion of categories of MRI findings for Cluster 12 containing $\mathbf{1 . 1 \%}$ of the $\mathbf{3 1 5 5}$ motion-segments. (a) The vertical bars on the graph represent the proportion of vertebral motion segments present within each coding category for each MRI finding. The bars on the graph do not show the 'Category 0', as that represents the presence of only normal findings. For pathologies with only one category, it represents the presence of the pathology. For 'disc signal intensity', 'disc height' and 'size of VESC', the categories refer to increasing severity or size of pathology with 'Category 1 ' being the least severe. For 'herniations', the categories represent the following types: $1=$ focal protrusion; 2 = broad-based protrusion; 3 =extrusion; 4 = sequestration. For 'type of VESC' the categories refer to the following types: $1=$ Type l; $2=$ Type II; $3=$ Type III; 4 = mixed Type I/II; $5=$ mixed Type II/III; $6=$ mixed Type I/III. For 'anterolisthesis' the categories refer to the Meyerdings classification. Detailed information on proportions of categories of MRI findings is described in Additional file 2. (b) The mean age of the motion segments was 37 years (SD 12) and the proportion of women was 41\%. (c) VESC = vertebral endplate signal change; Cat. = categories; EP = endplate.

\section{Discussion}

In this study, comprehensive MRI findings of lumbar vertebral motion segments were grouped using Latent Class Analysis into twelve clusters. Using content analysis, the identified clusters were then qualitatively grouped into five different biological pathways of degeneration that appear to have face validity.

This data-driven approach of the analysis of MRI findings is novel in LBP and our proof-of-concept study shows that this methodological approach produces plausible results suitable for further investigation. Two previous studies on LBP have used a multivariable approach to exploring the relationship between MRI findings and clinical variables, but in contrast to the current study, their multivariable analysis clustered the clinical variables and related these to single MRI findings. Takatalo et al. [27,28] used Latent Class Analyses to group multiple clinical variables (including pain, activity limitation and care seeking) from 468 people into five clusters which were then investigated for their association with individual MRI findings. It is not yet known whether the most revealing investigations of the association between MRI findings and clinical characteristics will involve the clustering of findings from both domains, only one domain or neither domain. In other areas of research, such as neurology and biological psychiatry, Latent Class Analysis has been used with MRI findings to investigate the validity of diagnostic subgroups or the accuracy of diagnostic tests [29-32].

Although the emphasis in the current study was on method, the identified MRI clusters appear to be biologically plausible and could be grouped into pathways of degeneration. These suggested pathways were based on the available evidence about the histological progression of disco-vertebral degeneration for different age groups [13] and on classification systems of degeneration based on histological [33] and MRI findings [19]. The chronology of the degenerative pathways was also informed by knowledge of the potential reversibility of some MRI findings (for example, an intervertebral disc herniation is potentially reversible but reduced disc height is not) and also, if the distribution of findings were mainly located in the lower or the upper lumbar motion segments. However, as there are few studies describing the precise association between the histology of degeneration and specific MRI findings, the evidence on which to base such grouping of MRI clusters is incomplete. Therefore, the clusters and degenerative pathways should be interpreted with caution and viewed as hypothesis-setting only.

MRIs of the lumbar spine can provide researchers with highly detailed information on spinal pathoanatomy, especially when quantified via comprehensive research protocols [21,22]. However, the volume of this detail can 


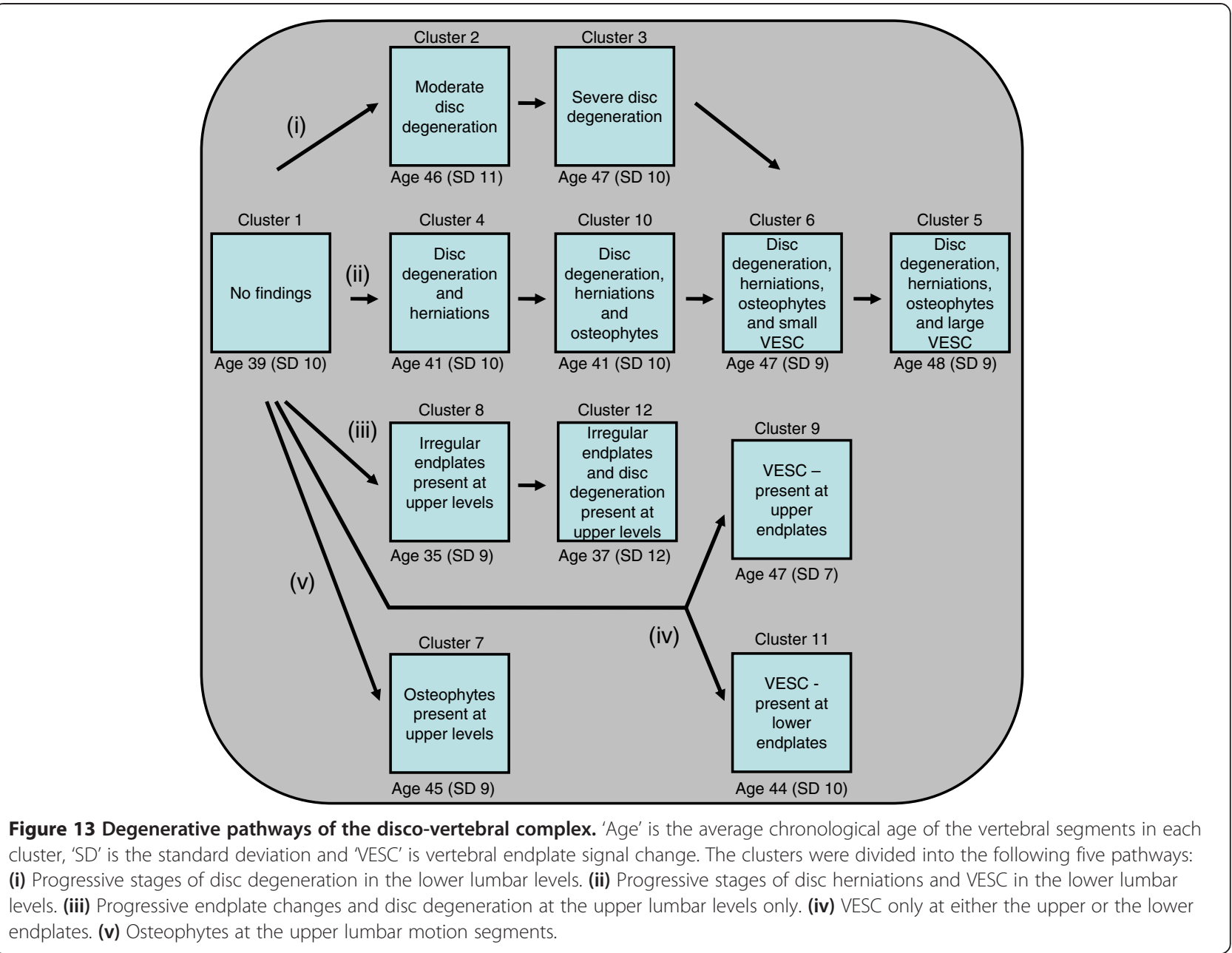

present a challenge for data analysis. For example, in our study, for each disco-vertebral level, there were more than 50 variables, each with up to seven response options. Traditional approaches to data reduction, such as regression analysis, can model the dominant pattern of association between MRI variables but do not accommodate the presence of multiple patterns that may reflect different stages of degeneration or different pathological pathways. Clustering techniques, such as Latent Class Analysis, have the advantage of allowing for that complexity.

We interpret these results as indicating that this research method should be further tested in different datasets to investigate if similar clusters are consistently replicated or if such clusters are highly sample-specific. If these clusters are reproducible, they could provide a platform from which to investigate associations between multivariate MRI findings and clinical findings, such as pain, activity limitations or other clinically relevant outcomes. Similarly, a further extension of this work would be to include MRI findings of structures other than the disco-vertebral complex, such as MRI findings of the vertebral canal, zygapophyseal joints and para-spinal soft tissue structures.

The strengths of this study are that a new statistical approach was applied to a large sample of MRI findings that had been rigorously described by an experienced research radiologist using a data extraction protocol with high reproducibility, and the results were cautiously interpreted. A weakness of the study is that its methodological focus means that the content of the identified clusters and biological pathways are preliminary and require extensive further study before their clinical validity, if any, can be determined.

\section{Conclusions}

This study has shown that Latent Class Analysis can be used to identify clusters of MRI findings from people with LBP and that those clusters can be grouped into biological pathways that have some face validity. If, when applied to other datasets of similar patients, this research method identifies reproducible clusters of MRI findings, these may form a stable platform to investigate the relationship 
between degenerative pathways and important clinical characteristics such as pain and activity limitation.

\section{Additional files}

Additional file 1: MRI findings and their distribution in the whole sample of vertebral segments. Detailed descriptive data of the MRI findings at a whole group level are tabulated in Additional file 1.

Additional file 2: The distribution (\%) of the MRI findings in the 12 clusters. Detailed descriptive data of the MRI findings at a cluster level are tabulated in Additional file 2 .

\section{Abbreviations}

LBP: Low back pain; MRI: Magnetic resonance imaging; SD: Standard deviation; VESC: Vertebral endplate signal change.

\section{Competing interests}

The authors declare that they have no competing interests.

\section{Authors' contributions}

RKJ, TSJ, PK and PMK all participated in conception and design of the project and in interpretation of the data. RKJ and PMK performed the analyses and wrote the draft manuscript. All authors have made substantial contributions to revising the manuscript. All authors read and approved the final manuscript.

\section{Acknowledgement}

The authors are grateful to The Foundation for Chiropractic Research and Postgraduate Education for funding this project. The authors thank Suzanne Capell, professional English language editor, for editing the manuscript and Joan Solgaard Sorensen, radiologist, M.D. for coding the MRI protocols.

\section{Author details}

${ }^{1}$ Research Department, Spine Centre of Southern Denmark, Hospital Lillebaelt, Middelfart, Denmark. ${ }^{2}$ Institute of Regional Health Services Research, University of Southern Denmark, Middelfart, Denmark. ${ }^{3}$ Institute of Sports Science and Clinical Biomechanics, University of Southern Denmark, Odense, Denmark.

Received: 10 September 2012 Accepted: 24 June 2013

Published: 1 July 2013

\section{References}

1. Hancock MJ, Maher CG, Laslett M, Hay E, Koes B: Discussion paper: what happened to the 'bio' in the bio-psycho-social model of low back pain. Eur Spine J 2011, 20:2105-2110.

2. Deyo RA, Rainville J, Kent DL: What can the history and physical examination tell us about low back pain? JAMA 1992, 268:760-765.

3. Kent $P$, Keating J: Do primary-care clinicians think that nonspecific low back pain is one condition. Spine (Phila Pa 1976) 2004, 29:1022-1031.

4. Fairbank J, Gwilym SE, France JC, Daffner SD, Dettori J, Hermsmeyer J, Andersson G: The role of classification of chronic low back pain. Spine (Phila Pa 1976) 2011, 36:S19-S42.

5. Kent $P$, Kjaer $P$ : The efficacy of targeted interventions for modifiable psychosocial risk factors of persistent nonspecific low back pain - A systematic review. Man Ther 2012, 17:385-401.

6. Donelson R, Aprill C, Medcalf R, Grant W: A prospective study of centralization of lumbar and referred pain. A predictor of symptomatic discs and anular competence. Spine (Phila Pa 1976) 1997, 22:1115-1122.

7. Long A, Donelson R, Fung T: Does it matter which exercise? A randomized control trial of exercise for low back pain. Spine (Phila Pa 1976) 2004, 29:2593-2602.

8. Boden SD, Davis DO, Dina TS, Patronas NJ, Wiesel SW: Abnormal magnetic-resonance scans of the lumbar spine in asymptomatic subjects. A prospective investigation. J Bone Joint Surg Am 1990, 72:403-408.

9. Jarvik JJ, Hollingworth W, Heagerty P, Haynor DR, Deyo RA: The Longitudinal Assessment of Imaging and Disability of the Back (LAIDBack) Study: baseline data. Spine (Phila Pa 1976) 2001, 26:1158-1166.
10. Jensen MC, Brant-Zawadzki MN, Obuchowski N, Modic MT, Malkasian D, Ross JS: Magnetic resonance imaging of the lumbar spine in people without back pain. N Engl J Med 1994, 331:69-73.

11. Jarvik JG, Hollingworth W, Martin B, Emerson SS, Gray DT, Overman S, Robinson D, Staiger T, Wessbecher F, Sullivan SD, Kreuter W, Deyo RA: Rapid magnetic resonance imaging vs radiographs for patients with low back pain: a randomized controlled trial. JAMA 2003, 289:2810-2818.

12. Chou D, Samartzis D, Bellabarba C, Patel A, Luk KD, Kisser JM, Skelly AC: Degenerative magnetic resonance imaging changes in patients with chronic low back pain: a systematic review. Spine (Phila Pa 1976) 2011, 36:S43-S53

13. Boos N, Weissbach S, Rohrbach H, Weiler C, Spratt KF, Nerlich AG: Classification of age-related changes in lumbar intervertebral discs: 2002 Volvo Award in basic science. Spine (Phila Pa 1976) 2002, 27:2631-2644.

14. Wang Y, Videman T, Battie MC: ISSLS Prize Winner: Lumbar Vertebral Endplate Lesions: Associations With Disc Degeneration and Back Pain History. Spine (Phila Pa 1976) 2012, 37:1490-1496.

15. Lusins JO, Cicoria AD, Goldsmith SJ: SPECT and lumbar MRI in back pain with emphasis on changes in end plates in association with disc degeneration. J Neuroimaging 1998, 8:78-82.

16. Schmid G, Witteler A, Willburger R, Kuhnen C, Jergas M, Koester O: Lumbar disk herniation: correlation of histologic findings with marrow signal intensity changes in vertebral endplates at MR imaging. Radiology 2004, 231:352-358.

17. van Dieen $\mathrm{JH}$, Weinans $\mathrm{H}$, Toussaint $\mathrm{HM}$ : Fractures of the lumbar vertebral endplate in the etiology of low back pain: a hypothesis on the causative role of spinal compression in aspecific low back pain. Med Hypotheses 1999, 53:246-252.

18. Cheung KM, Karppinen J, Chan D, Ho DW, Song YQ, Sham P, Cheah KS, Leong JC, Luk KD: Prevalence and pattern of lumbar magnetic resonance imaging changes in a population study of one thousand forty-three individuals. Spine (Phila Pa 1976) 2009, 34:934-940.

19. Pfirrmann CW, Metzdorf A, Zanetti M, Hodler J, Boos N: Magnetic resonance classification of lumbar intervertebral disc degeneration. Spine (Phila Pa 1976) 2001, 26:1873-1878.

20. Jensen RK, Leboeuf-Yde C, Wedderkopp N, Sorensen JS, Manniche C: Rest versus exercise as treatment for patients with low back pain and Modic changes. A randomized controlled clinical trial. BMC Med 2012, 10:22.

21. Jensen TS, Sorensen JS, Kjaer P: Intra- and interobserver reproducibility of vertebral endplate signal (modic) changes in the lumbar spine: the Nordic Modic Consensus Group classification. Acta Radiol 2007, 48:748-754

22. Solgaard SJ, Kjaer P, Jensen ST, Andersen P: Low-field magnetic resonance imaging of the lumbar spine: reliability of qualitative evaluation of disc and muscle parameters. Acta Radiol 2006, 47:947-953.

23. Meyerding HW: Low back ache and sciatic pain associated with spondylolisthesis and protruded intervertebral disc. J Bone Joint Surg Am 1941, 23:461.

24. Magidson J, Vermunt JK: Latent class models for clustering: A comparison with K-means. Canadian Journal of Marketing Research 2002, 20:37-44.

25. Wallace CS, Dowe DL: Intrinsic classification by MML - the SNOB program, Proc 7th Australian Joint Conference on Artificial Intelligence. Armidale NSW, Australia: World Scientific; 1994:37-44.

26. Wallace CS: Statistical and inductive inference by minimum message length. New York, USA: Springer; 2005

27. Takatalo J, Karppinen J, Niinimaki J, Taimela S, Nayha S, Mutanen P. Sequeiros RB, Kyllönen E, Tervonen O: Does lumbar disc degeneration on magnetic resonance imaging associate with low back symptom severity in young Finnish adults. Spine (Phila Pa 1976) 2011, 36:2180-2189.

28. Takatalo J, Karppinen J, Niinimaki J, Taimela S, Mutanen P, Sequeiros RB, Näyhä S, Järvelin MR, Kyllönen E, Tervonen O: Association of Modic Changes, Schmorl's Nodes, Spondylolytic Defects, High-Intensity Zone Lesions, Disc Herniations, and Radial Tears With Low Back Symptom Severity Among Young Finnish Adults. Spine (Phila Pa 1976) 2012, 37:1231-1239.

29. Prust M, Wang J, Morizono H, Messing A, Brenner M, Gordon E, Hartka T, Sokohl A, Schiffmann R, Gordish-Dressman H, Albin R, Amartino H, Brockman K, Dinopoulos A, Dotti MT, Fain D, Fernandez R, Ferreira J, Fleming J, Gill D, Griebel M, Heilstedt H, Kaplan P, Lewis D, Nakagawa M, Pedersen R, Reddy A, Sawaishi Y, Schneider M, Sherr E, Takiyama Y, Wakabayashi K, Gorospe JR, Vanderver A: GFAP mutations, age at onset, and clinical subtypes in Alexander disease. Neurology 2011, 77:1287-1294. 
30. Wolz R, Julkunen V, Koikkalainen J, Niskanen E, Zhang DP, Rueckert D, Soininen H, Lötjönen J: Alzheimer's Disease Neuroimaging Initiative: Multi-method analysis of MRI images in early diagnostics of Alzheimer's disease. PLoS One 2011, 6:e25446.

31. Yoon JH, Tamir D, Minzenberg MJ, Ragland JD, Ursu S, Carter CS: Multivariate pattern analysis of functional magnetic resonance imaging data reveals deficits in distributed representations in schizophrenia. Biol Psychiatry 2008, 64:1035-1041.

32. Zwemmer JN, Berkhof J, Castelijns JA, Barkhof F, Polman CH, Uitdehaag BM: Classification of multiple sclerosis patients by latent class analysis of magnetic resonance imaging characteristics. Mult Scler 2006, 12:565-572

33. Thompson JP, Pearce RH, Schechter MT, Adams ME, Tsang IK, Bishop PB:

Preliminary evaluation of a scheme for grading the gross morphology of the human intervertebral disc. Spine (Phila Pa 1976) 1990, 15:411-415.

doi:10.1186/1471-2474-14-198

Cite this article as: Jensen et al:: Can pathoanatomical pathways of

degeneration in lumbar motion segments be identified by clustering MRI findings. BMC Musculoskeletal Disorders 2013 14:198.

\section{Submit your next manuscript to BioMed Central and take full advantage of:}

- Convenient online submission

- Thorough peer review

- No space constraints or color figure charges

- Immediate publication on acceptance

- Inclusion in PubMed, CAS, Scopus and Google Scholar

- Research which is freely available for redistribution 